\title{
Pharmacist involvement in care transitions in an academic medical center
}

\author{
Abstract \\ Background: Care transitions pose increased risks for medication errors. Pharmacists \\ are uniquely suited to provide assistance with medication reconciliations and medication \\ counseling during care transitions.
}

Objective: The primary objective of this study was to evaluate the impact of a pharmacist reviewing discharge medication reconciliations and providing medication counseling at the time of hospital discharge.

Methods: This retrospective chart review includes adult patients discharged from a range of medicine services from August 23, 2014 to February 7, 2015, at a large academic medical center. The primary outcome was 30 -day hospital readmission rate, and secondary outcomes included time-to-hospital readmission, frequency of medication errors, type of medication errors, and frequency of errors per pharmacologic class.

Results: In the final analysis, 175 patients were included. The majority of patients in the intervention group had at least one medication error, and the most common error occurred with cardiovascular agents. There was no statistically significant difference between the two groups regarding 30-day hospital readmissions.

Conclusion: Although the findings did not show significant differences, a pharmacist was able to identify medication errors in most patients. Additionally, a lack of difference in hospital readmissions is clinically significant, given the intervention group had higher acuity of health care needs.

Keywords: transitions of care, medication safety internal medicine
Volume 5 Issue I - 2017

\author{
Meredith Ann Sigler,' Amy Nicole Thompson ${ }^{2}$ \\ 'Texas Tech University Health Sciences Center School of \\ Pharmacy, USA \\ ${ }^{2}$ College of Pharmacy, University of Michigan, USA
}

\begin{abstract}
Correspondence: Meredith Ann Sigler, Assistant Professor of Pharmacy Practice, Division of Ambulatory Care, Texas Tech University Health Sciences Center School of Pharmacy, 4500 S Lancaster Rd, BIdg 7, R\#I I9A, Dallas, TX 75216, USA, Tel (214) 358-906I, Email meredith.sigler@ttuhsc.edu
\end{abstract}

Received: August 22, 2016 | Published: June 09, 2017

\section{Introduction}

Transitions of care can be a confusing and challenging experience for patients, as many of their medications may change during these times. The National Transitions of Care Coalition defines transitions of care as the movement of a patient from one health care setting to another, or to a patient's home. ${ }^{1}$ One aspect of the care transition is reconciling a patient's entire medication regimen before an inpatient admission with their discharge regimen. ${ }^{2}$ Providing an accurate discharge medication reconciliation is important for minimizing harm on return to home, reducing therapeutic duplications, and reducing patient morbidity and re-hospitalization. The importance of medication reconciliations are demonstrated by these activities being considered a Joint Commission National Patient Safety Goal and a part of the Centers for Medicare and Medicaid Services Meaningful Use criteria. ${ }^{3,4}$

Medication reconciliations are a critical step for identifying potential medication errors. In a 2009 study by Jack and colleagues, medication reconciliations were completed by pharmacists via a telephone call 2 to 4 days after being discharged from a hospital, which found 126 medication errors out of 195 medication reconciliations completed. Out of these errors, $53 \%$ needed corrective action. ${ }^{5}$ Errors found when conducting medication reconciliations may have the potential to influence 30 -day hospital readmission rates. The likelihood of 30-day hospital readmission after instituting a collaborative pharmacist care transitions program was evaluated in a retrospective cohort study. Patients in the intervention group received bedside delivery of medications and a phone follow-up by a pharmacist to review medications. The odds of 30-day readmission for the patients not receiving the intervention was 1.9 higher than those who had pharmacy involvement at discharge. ${ }^{6}$ Furthermore, in a prospective study by Vira and colleagues, pharmacists conducted admission and discharge medication reconciliations for patients in the acute care units in a Canadian community hospital. Out of 60 patients, $60 \%$ had at least one medication discrepancy and $51 \%$ of the patients did not receive discharge instructions.

A 2013 single-center, observational study evaluated 517 patients to determine the incidence of unintended admission medication discrepancies that were resolved by a pharmacist. Patients were identified for inclusion in the study if they were identified as highrisk for 30-day hospital readmission. Medication discrepancies were found in $25 \%$ of the patients, with $46 \%$ of the discrepancies being considered significant or serious. The program developed by this study is estimated to have a net cost savings value of $\$ 5.7$ million over 5years. ${ }^{8}$

Based on the significant findings of prior research with medication reconciliation processes, the purpose of this study was to evaluate whether a pharmacists assisting with medication reconciliation and providing medication counseling at the time of discharge would reduce the 30-day hospital readmission rate in adult patients discharged from a large academic medical center. Other criteria investigated included time-to-hospital readmission, number of medication errors identified, and pharmacologic class in which the error was made.

\section{Methods}

The intervention, assessed through a retrospective chart review, included a pharmacist reviewing patients' discharge medication reconciliations for errors and appropriateness as well as conducting 
discharge medication counseling. Once an error was identified, the pharmacist contacted the ordering provider and documented the encounter in the electronic medical record. Adults patients, 18 years or older, discharged from a southern United States academic medical center from August 23, 2014 to February 7, 2015 were included. Patients who were discharged from one of the following services were included in the analysis: family medicine; general medicine; hospitalists; or neurology. Patients included in the intervention group were limited to those discharged during an average of 32 working hours per month, as this was the timeframe during which the standardized intervention of medication reconciliation reviews and discharge counseling were provided at the institution. Patients in the control group were limited to the same average of 32 hours per month for comparison purposes. Exclusion criteria included: pregnant females; patients readmitted within 30days for a planned procedure; patients receiving counseling from a pharmacist at time of admission and/ or for a specific medication; patients being discharged to hospice; patients leaving against medical advice; and patients with charts noting that the patient had died within 30days of their index discharge.

Excel ${ }^{\circledR}$ (version 2013, Microsoft, Redmond, WA) and SPSS (version 22, IBM, Armonk, NY) were utilized for data entry and analysis. Baseline characteristics were analyzed by descriptive statistics, utilizing percentages and means for categorical and continuous variables, respectively. Pearson $X 2$ and Fisher's exact test, when sample sizes were small, were employed for the determination of the primary outcome. Secondary outcomes were analyzed by descriptive statistics. Time-to-readmission was analyzed by Cox proportional hazards regression to determine if there was a difference between the intervention and control group, adjusting for whether patients were identified as high-risk or not.

Data for analysis were obtained from an electronic chart review. The following data were gathered for investigative purposes: age; gender; discharge service line; admitting diagnosis; number of discharge prescriptions; whether the patient had been admitted within the past year; length of hospitalization; type of medication error; whether the patient was considered high-risk; whether the patient was on 10 or more chronic medications; pharmacologic class in which the medication error occurred, in the intervention group; whether a medication error occurred as noted in the intervention group; total number of medication errors in the intervention group; whether the patient was readmitted within 3 days; time to hospital readmission within 90days; and whether the readmission was related to a medication. High-risk patients were defined as patients having at least one of the following: hospital readmission within the previous 30days; diagnosis of acute pneumonia, hospitalized for acute myocardial infarction, heart failure, or chronic obstructive pulmonary disease; more than 10 scheduled maintenance prescription medications; medication order for an anticoagulant, dofetilide, clozapine, highly active antiretroviral therapy, tacrolimus, cyclosporine, or lithium. The definition of high-risk was used throughout the institution as a means to categorize patients' health care acuity at the time of admission.

If a medication error was identified, the ordering provider was notified. The types of medication errors included contraindication, commission, omission, ordering error, and wrong dose and/ or frequency. Other potential errors included prescriptions being sent to a pharmacy that was unavailable to the patient at time of discharge, and lack of appropriate follow-up. The primary outcome was the 30-day hospital readmission rate, and secondary outcomes included time to hospital readmission, number of medication errors, type of error identified, and frequency of errors related to the pharmacologic classes.

\section{Results}

There were 398 patients assessed for eligibility in the analysis and data were collected on 175 patients. Reasons for exclusions are presented in Figure 1. The majority of patients excluded were discharged from a service other than that stated a priori. Other patients were excluded because a pharmacist conducted an admission medication reconciliation or for counseling on one specific medication $(6.78 \%)$. Other reasons for exclusion included pregnant patients $(0.50 \%)$, patients not discharged on date specified $(0.75 \%)$, patients discharged against medical advice $(0.25 \%)$, patients who died within 30 days of index discharge date (3.27\%), and patients readmitted due to a planned procedure $(2.26 \%)$.

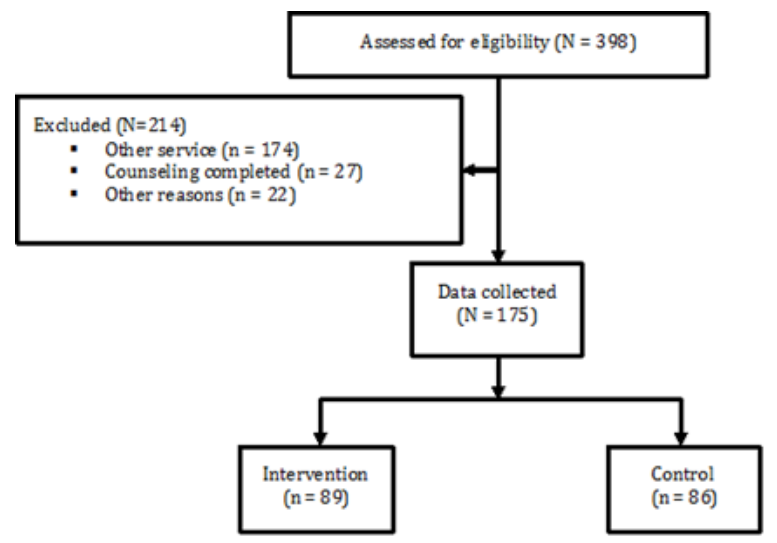

Figure I Diagram of patient selection.

The majority of patients were between 50 and 55years of age, female, had an average of least 1 prior admission in the past year, and had an average length of stay between 5 and 8 days. Observable differences between the intervention group compared to the control group included more patients in the intervention group were admitted due to an infection and more patients in the control group were admitted for a neurologic abnormality. The intervention group had a greater number of new discharge prescriptions and were on 10 or more chronic medications. Finally, more patients in the intervention group were considered high-risk patients (Table 1 for baseline characteristics).

With respect to the primary outcome, Table 2 summarizes the 30 day readmission rates for the two groups. There was no statistically significant difference observed. Sub-cohort results of the intervention group are shown in Table 3. The majority of patients in the intervention group had an error detected at the time of discharge $(76.4 \%$ of patients). Withal, $9.0 \%$ of patients in the intervention group had their prescriptions at discharge sent to a The most common error type that occurred was an ordering error (Figure 2 for types of errors). Few errors existed due to a contraindication of the medication. Cardiology was the pharmacologic class which had the most numerous errors, followed by Endocrinology. Notably, there were a total of 7 errors identified regarding anticoagulation regimens (Figure 3 for errors in pharmacologic classes). Regarding time-to-readmission, there were a total of 43 patients in both groups readmitted within 90 days (Figure 4). There was no difference in time-to-readmission between the intervention group compared to the control group $(P>0.9)$. Patients that were identified as high-risk did had a higher likelihood of readmission compared to patients that were not high-risk $(\mathrm{HR}=2.2$; $95 \%$ CI: 1.1 to $4.4 ; P=0.02$ ). 
Table I Baseline Characteristics

\begin{tabular}{|c|c|c|c|}
\hline Characteristic & Intervention $(n=89)$ & Control $(n=86)$ & p-value (95\% Confidence interval) \\
\hline Age mean (SD) & $54.8( \pm 18.4)$ & $49.8( \pm 17.7)$ & $0.68(-10.4$ to 0.376$)$ \\
\hline Male, n (\%) & $38(42.7)$ & $35(40.7)$ & 0.072 \\
\hline Length of stay Mean (SD) & $7.83( \pm 17.6)$ & $5.34( \pm 3.79)$ & $0.2(-6.32$ to 1.34$)$ \\
\hline Admissions in past year Mean (SD) & $1.46( \pm 2.12)$ & $\mathrm{I} .09( \pm \mathrm{I} .7 \mathrm{I})$ & $0.209(-0.943$ to 0.208$)$ \\
\hline Discharge services & & & $p$-Value \\
\hline General Medicine n (\%) & $44(49.4)$ & $26(30.2)$ & \multirow{4}{*}{$<0.001$} \\
\hline Hospitalist n (\%) & $24(27.0)$ & $24(27.9)$ & \\
\hline Neurology n (\%) & $7(7.90)$ & $34(39.5)$ & \\
\hline Family Medicine $\mathrm{n}(\%)$ & $14(15.7)$ & $2(2.3)$ & \\
\hline Admission diagnosis groups & & & $p$-Value \\
\hline Endocrine $\mathrm{n}(\%)$ & $10(11.2)$ & $3(3.60)$ & 0.06 \\
\hline Psychiatry n (\%) & $0(0.00)$ & $7(7.87)$ & 0.006 \\
\hline Gastrointestinal n (\%) & $8(9.00)$ & II (I2.8) & 0.653 \\
\hline Neuro n (\%) & $14(15.7)$ & $29(33.7)$ & 0.006 \\
\hline Hematology n (\%) & II (12.4) & $9(10.5)$ & 0.694 \\
\hline Pulmonary n (\%) & $15(16.9)$ & $13(15.1)$ & 0.754 \\
\hline Infectious $\mathrm{n}(\%)$ & $18(20.2)$ & II (I2.8) & 0.186 \\
\hline Dermatology n (\%) & $3(3.4)$ & $\mathrm{I}(\mathrm{I} .20)$ & 0.329 \\
\hline Renal/Urology n (\%) & $3(3.4)$ & $2(2.30)$ & 0.678 \\
\hline Other n (\%) & $5(5.60)$ & $3(3.50)$ & 0.5 \\
\hline Number of new prescriptions, mean (SD) & $2.84( \pm 2.44)$ & $1.34( \pm 1.96)$ & $<0.00$ I $(-2.17$ to -0.843$)$ \\
\hline High Risk, n (\%) & $57(64.0)$ & $23(26.7)$ & $<0.001$ \\
\hline Patients on $>10$ chronic medications, $\mathrm{n}(\%)$ & $33(37.1)$ & $16(18.6)$ & 0.007 \\
\hline
\end{tabular}

Table 2 30-day Hospital Readmission Rates

\begin{tabular}{llll}
\hline 30-day Readmission & Intervention $(\mathbf{n}=\mathbf{8 9})$ & Control $(\mathbf{n}=\mathbf{8 6})$ & $\mathbf{p}$-Value \\
\hline $\mathrm{n}(\%)$ & $9(10.1)$ & $7(8.1)$ & $0.65 \mathrm{I}$ \\
\hline
\end{tabular}

Table 3 Sub-cohort Intervention Errors

\begin{tabular}{ll}
\hline Intervention error & \\
\hline Total number of errors, $\mathrm{n}(\%)^{\mathrm{a}}$ & $\mathrm{I} 04(76.4)$ \\
Average number of errors, mean (SD) & $\mathrm{I} .18( \pm \mathrm{I} . \mathrm{I3})$ \\
Prescription sent to closed, pharmacy n (\%) & $8(9.00)$ \\
\hline
\end{tabular}

aPercent calculated based on how many patients had at least one medication error.

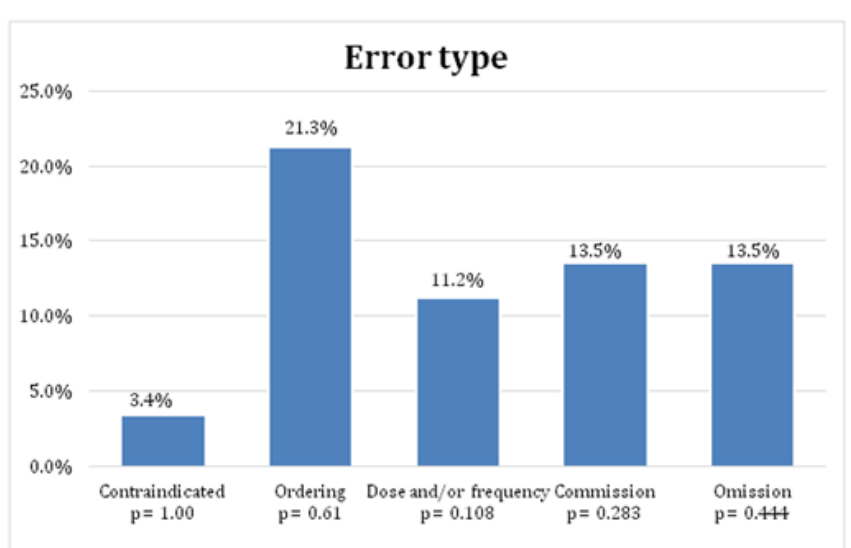

Figure 2 Type of error identified in the intervention cohort.

\section{Discussion}

No difference was found in 30-day hospital readmission rates between the intervention and control group. Regardless, the majority of patients in the intervention group had at least one medication error detected at the time of discharge. As the intervention cohort contained a higher-acuity and higher-risk of hospital readmission group, the lack of a difference could be considered clinically significant. However, a validated risk stratification should be developed to be able to appropriately assess any clinically important differences.

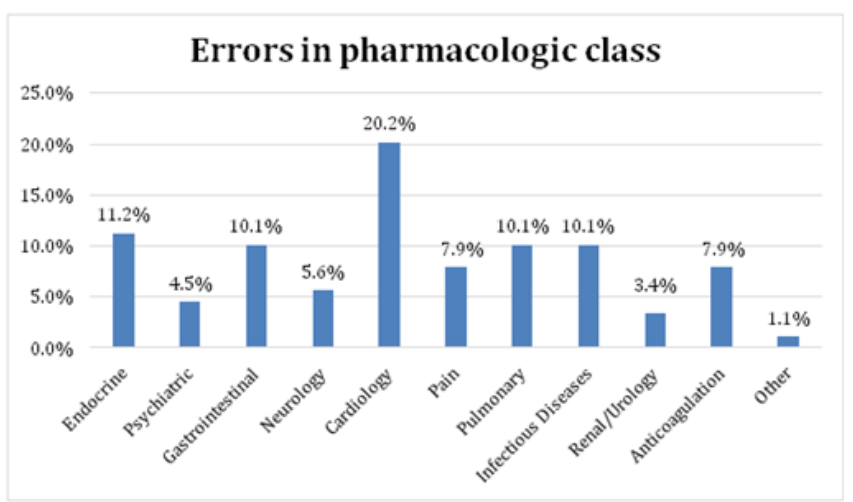

Figure 3 Frequency of errors in pharmacologic classes.

Care transitions present the potential for patients to experience a gap in their medical care. Medication errors at the time of discharge as well as patients inappropriately using their medications due to lack of understanding of their medication regimen contribute to possible 
hospital readmissions. The National Care Coalition notes that care transitions should be, "well planned and appropriately timed," in an effort to prevent such errors from occurring.1 Pharmacists can make influential interventions to assist in care transitions by assessing discharge medication regimens for appropriateness and providing medication counseling. However, a national survey by Kern and colleagues found that, out of 393 respondents, $70 \%$ of pharmacists spend less than $10 \%$ of a 40 hour work week assisting with care transitions activities. ${ }^{9}$

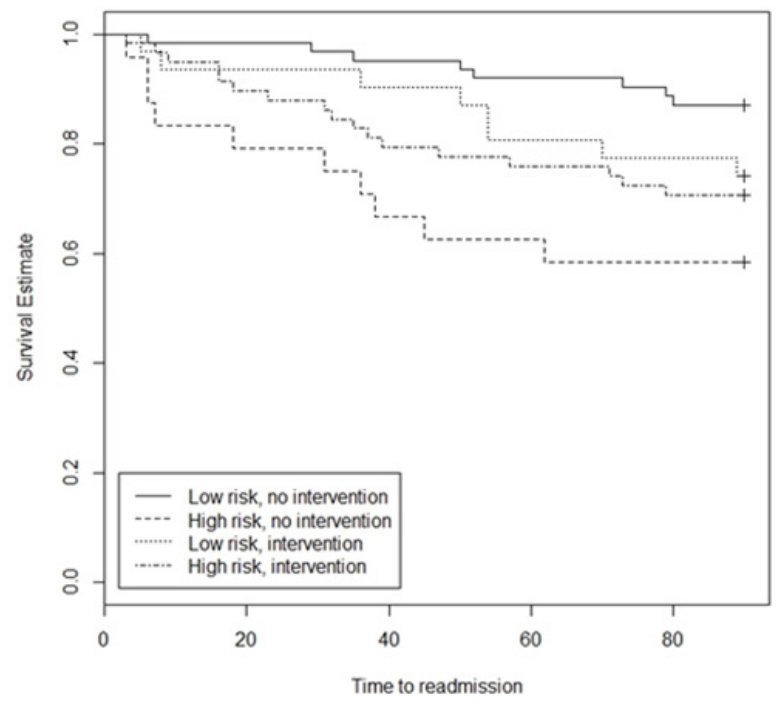

Figure 4 Time-to-readmission.

A Cox proportional hazards regression model was used to determine if intervention group impacted time to readmission after adjusting for whether a patient was high risk or not. Intervention was not significantly related to time to readmission $(p>0.9)$. Patients that were high risk had a higher risk of readmission compared to those that were low risk $(\mathrm{HR}=2.2,95 \% \mathrm{Cl}$ : I.I-4.4; $\mathrm{p}=0.02$ ).

In an effort to better utilize resources and focus on patients with a perceivably higher risk of hospital readmission, some institutions are creating criteria to identify such patients for pharmacists to conduct medication interventions. In the previously mentioned 2013 observational study assessing admission medication reconciliations, only high-risk patients had this service provided. The authors' definition for high-risk included the following criteria: hospitalized in the previous 30days; primary diagnosis of heart failure or acute myocardial infarction; active medication orders for warfarin, concomitant orders for aspirin and clopidogrel; past medical history of heart failure or myocardial infarction; or 2 or more diagnoses of end-stage renal disease, diabetes mellitus, hypertension, or chronic obstructive pulmonary disease. 8 These criteria are similar to the ones utilized for our institution's definition of a high-risk patient (see Methods for full list). An important limitation for the use of these criterion includes assessment only at the time of admission. It may prove prudent to conduct an ongoing assessment of a patient's risk for hospital readmission throughout their hospitalization, as their status could drastically change from admission to discharge.

This study was conducted at a large academic medical center, serving a sizeable patient population with a wide range of medical conditions. As this study was limited to only one institution, the findings are not generalizable to all hospitals. While ordering errors were common during the duration of the study, this could partly be explained by the introduction of a new electronic medical record system being utilized starting July 1, 2014. Other notable limitations include the fact that not all readmissions could be accounted for as patients could have presented to outside facilities. Additionally, the intervention group only had services provided on a limited basis, accounting for the small sample size. It is unknown if there are higher rates of errors on the weekend versus normal working hours, which could have impacted the results of the this study given the limited availability of the standardized intervention. Because the sample size was small, this study lacks adequate power to test whether a difference exists. The acceptance rate of the intervention group recommendations made to the physicians are unknown, as this was unable to be detected retrospectively. Due to the retrospective design, errors on discharge summaries, evaluation of appropriate follow-up, and appropriate patient comprehension at time of medication counseling were unable to be determined. Other errors such as cost prohibition in patients' affordability of their regimen and analysis for potential differences of health literacy between the two groups were also not recorded.

\section{Conclusion}

Having a pharmacist review patients' discharge medication reconciliations for appropriateness and provide medication counseling did not reduce 30 -day hospital readmission rates. There were several medication errors identified during this process and 30day hospital readmissions were equivalent despite the higher-acuity patients in the intervention group. These findings may lead to more involvement of the pharmacy staff at the time of care transitions, focusing on prevention of possible medication errors and assessing for appropriateness of the medication regimen as the patient transfers to a new healthcare setting or as the patient is discharged from the hospital. Future efforts should focus on assessing both admission and discharge medication regimens for appropriateness based on patientspecific factors, including ensuring medications are discontinued at discharge, and this information is reliably sent to the patients' outpatient pharmacies.

\section{Acknowledgments}

None.

\section{Conflicts of interest}

The authors declare no conflicts of interest related to this article.

\section{Funding}

None.

\section{References}

1. http://www.ntocc.org/portals/0/TransitionsOfCare_Measures.pdf

2. http://psnet.ahrq.gov/primer.aspx?primerID $=1$

3. http://www.jointcommission.org/assets/1/6/2015_NPSG_AHC1.PDF

4. http://www.healthit.gov/providers-professionals/achieve-meaningfuluse/core-measures-2/medication-reconciliation

5. Jack BW, Chetty VK, Anthony D, et al. A reengineered hospital discharge program to decrease rehospitalization. Ann Intern Med. 2009;150(3):178-187.

6. Kirkham HS, Clark BL, Paynter J, et al. The effect of a collaborative pharmacist-hospital care transition program on the likelihood of 30-day readmission. Am J Health-Syst Pharm. 2004;71(9):739-745.

7. Vira T, Colquhoun M, Etchells E. Reconcilable differences: correcting medication errors at hospital admission and discharge. Qual Saf Health Care. 2006;15(2):122-126. 
8. Buckley MS, Harinstein LM, Clark KB, et al. Impact of a clinical pharmacy admission medication reconciliation program on medication errors in "high-risk" patients. Ann Pharmacother. 2013;47(12):15991610 .
9. Kern KA, Kalus JS, Bush C, et al. Variations in pharmacy-based transition-of-care activities in the United States: a national survey. Am J Health-Syst Pharm. 2014;71(8):648-656. 